\title{
Characteristics of balance performance in the Chinese elderly by age and gender
}

Hongmei $\mathrm{Wu}^{1 \dagger}$, Yifan Wei $^{1 \dagger}$, Xiangqi Miao ${ }^{1 \dagger}$, Xia Li$^{2}$, Yang Feng ${ }^{1,3}$, Zhenzhen Yuan ${ }^{1,4}$, Peng Zhou ${ }^{5}$, Xiaolei Ye Jianhong Zhu' ${ }^{1}, Y u$ Jiang $^{5^{*}}$ and Qinghua Xia ${ }^{5^{*}}$

\begin{abstract}
Background: Population aging has been an emerging public and health concern globally. Balance performance can be applied as an indicator of functional status and a predictor of health outcomes in the elderly. However, reference data of balance performance in the elderly generated from large scale studies have been very limited. In research and geriatric assessment settings, the age and gender specific data on balance performance are indispensable prerequisites for identifying subpopulation with and at risk of impairments and subsequently implementing targeted interventions in clinics and public health to improve their balance performance.
\end{abstract}

Methods: A total of 1984 elderly subjects aged 60 to 97 years from community settings in urban China were investigated. The balance performances together with 3 individual domains and 16 items were evaluated using the X16 balance testing scale.

Results: In the elderly, with age increases each item, individual domain, and overall balance performance scores decreased gradually. Meanwhile, individual variations of individual domains and overall balance performance were all increased over age. Relative to levels of 60-years, postural stability and overall balance performance decreased significantly since 65 years old, static balance and dynamic balance capacities started to decrease significantly since 70 years old. There was no significant difference in each balance domain and overall balance performance between men and women. Across age groups, portions of individuals able to perform task 4, 8 and 11 successfully were the lowest amongst their corresponding domains static balance, postural stability, and dynamic balance, respectively. Similar patterns were observed in both men and women. Balance performances were categorized into poor, fair, and good groups with scores of 0 to 10,11 to 17 , and 18 to 20, respectively. With increases of age, proportions with poor and fair balance capacities elevated stably.

Conclusions: In the elderly, with advances in age, abilities of overall balance performance, individual domains of static balance, postural stability, and dynamic balance, and successful performances on specific tasks declined gradually and stably. The deterioration started to be obvious since 65-75 years. Men and women had similar patterns.

Keywords: Elderly, Balance, Age, Gender, Static balance, Postural stability, Dynamic balance

*Correspondence: jiangyukx@126.com; xiaqinghua56@126.com ${ }^{\dagger}$ Hongmei Wu, Yifan Wei, and Xiangqi Miao contributed equally to this work.

${ }^{5}$ Changning Center for Disease Control and Prevention, Shanghai 200051, People's Republic of China

Full list of author information is available at the end of the article

\section{Background}

The pace of population aging around the world has been increasing progressively and rapidly for decades. Global population aged 65 years and over were 151.1 million in 1960, 328.2 million in 1990, and 702.9 million in 2019 , accounted for $4.98 \%, 6.16 \%$, and $9.11 \%$, respectively, indicating a double of the elderly population every 30 years in 
number and accelerating increase in proportion. Nearly all countries over the world have been experiencing growths in the number and proportion of the elderly in their population. In China, the population aged 65 years and over were 24.4 million in 1960, 66.3 million in 1990, and 164.5 million in 2019 , accounted for $3.69 \%$, 5.63\%, and $11.47 \%$ of the total population, respectively, showing higher increasing rate in contrast to the pace worldwide. Over the upcoming decade, numbers of global and Chinese population aged 65 or over will project to 997.5 and 247.0 million, which will account for $11.67 \%$ and $16.87 \%$ of their total population, respectively [1].

Population aging is a triumph of development, which is mainly attributable to improved nutrition, sanitation, medical advances, health care, education and economic wellbeing. However, it comes together with challenges to individuals, families, societies and the global community in terms of health status [2]. With increases of age in the elderly, gradual declines in physiological and functional conditions are predictable, consequently their health statuses have visibly deteriorated.

Balance performance has been considered as an indicator of health status in the elderly. The ability of maintaining balance is one imperative component part in carrying out most daily activities independently and successfully $[3,4]$.

There is no universally accepted definition for human balance, balance herein is described as the ability to maintain various positions and achieve or restore the state of postural equilibrium during activities. For example, the maintenance of specified postures such as sitting or standing, automatic responses to voluntary body or extremity movements such as movements between postures, and reactions to external disturbances [5-7]. Balance can be classified roughly into static balance and dynamic balance. Static balance refers to abilities to maintain a steady position in sitting or standing on a fixed, firm, unmoving base of support. Dynamic balance is abilities to maintain or regain the center of mass within the base of support when the body is moving $[5,8]$.

A great number of studies showed that balance performance in the elderly declined in contrast to the young adults and middle-aged adults, and among the elderly balance abilities deteriorated with advances in age [915]. Previous investigations evaluated balance performance with various approaches, such as functional base of support, functional reach test, the timed up and go test, static balance, etc. However, reference data of balance performance in the elderly by age and gender generated from large scale studies have still been lacking.

Mini-Balance Evaluation Systems Test (Mini-BESTest) offers a unique, brief clinical rating scale for dynamic balance. It helps in directing the specific treatments for the patients and identifying the specific system affected and change with therapy. But it requires equipments and expertised raters, also it needs $10-15 \mathrm{~min}$ to administer, and longer depending upon the severity of conditions, which make it may not be practical for regular use in community settings for large scaled screening or evaluation [16].

The Berg Balance Scale (BBS) is the most commonly used assessment tool for stroke rehabilitation in the clinic and has been frequently applied to identify and evaluate balance impairment in the elderly. The scoring system of the BBS is subtle, each item is scored on a scale from 0 to 4 , and the differentiation from points 1 to 3 requires careful attention from the investigators. Meanwhile, this scale takes 15 to $20 \mathrm{~min}$ to complete. Which makes BBS not suitable for large scaled screening in community settings [6].

The X16 balance testing scale for the elderly was designated for use in community setting and large scale screening. One significant strength of the X16 scale was it was practical and reliable for use in assessments of overall balance performance and individual domains including static balance, postural stability, and dynamic balance simultaneously [17]. In present study a total of 1984 elderly subjects in the unique community context of urban China were investigated and their balance performances were evaluated using the X16 scale. This study established normative data, specified variations between age groups, recognized differences between genders, identified high risk subpopulation at overall balance, individual domain, and specific task levels, and provided guidances on further examination and intervention measures at clinics and public health levels.

\section{Methods}

\section{Subjects}

The project was approved by Institutional Review Board (IRB) of Changning Center for Disease Control and Prevention, Shanghai. The written informed consents were obtained from all of the participants. All protocols for involving humans were in accordance to guidelines of institutional declaration.

The inclusion criteria included, residents lived at home alone or with family in Shanghai for 6 months or longer per year, men and women, aged 60 years or over, functionally independent, physically active, able to ambulate without assistance from others or assistive devices, and able to understand and answer the interview questions. The exclusion criteria included, living in a nursing home, hospitalization, dementia, visual deficits, unable to finish the test for other health reasons.

The subjects were recruited from Changning district of Shanghai, China. From which 8 resident communities 
were randomly selected, and 375 families were randomly selected from each community. A total of 3000 families were investigated and there were 2312 individuals aged 60 years or above. All of them were assessed for eligibility, of which 168 individuals were incapable of walking independently and unable to complete the balance test, 92 individuals had cognitive function impairments being unable to understand the study, and 68 individuals had incomplete information, thus 328 individuals were excluded. Finally 1984 subjects were included for analysis.

\section{Data collection}

Data were collected through a face-to-face interview by trained investigators. Balance performance was examined on-site using X16 balance testing scale for the elderly [17]. Domain I is static balance, domain II is postural stability, and domain III is dynamic balance. Items are named as domain number followed by item number, for example, I 2 indicates the item 2 which is in domain I, II 7 indicates the item 7 which is in domain II. Items were numbered consecutively through the whole balance testing scale. Each item is scored from 0 to 1 or 2 points. Zero point indicates the impairment, and the 1 or 2 points indicates independence. The scores for the static balance, postural stability, and dynamic balance domains are ranged from 0 to 4,0 to 8 , and 0 to 8 points, respectively. The total score for balance performance is ranged from 0 to 20 points (Table S1).

\section{Statistical analyses}

EpiData 3.0 (The EpiData Association, Odense, Denmark) was used for data entry, and SPSS 22.0 (SPSS Inc. Chicago, IL, USA) was applied for data processing and statistical analysis.
The data of balance performance scores were expressed as mean \pm standard deviation (SD), differences between age groups were analyzed with one-way analysis of variance (ANOVA) followed by Tamhane's T2 for multiple comparisons, differences between men and women were compared with Student's $t$ test. The categorical data between age groups or between items were analyzed with Chi-squared test followed by Bonferroni correction for multiple comparisons. Balance performances were categorized into groups with Two-Step Cluster Analysis. The significance level was set at 0.05 .

\section{Results}

\section{Age and gender compositions of the elderly}

A total of 1984 participants were recruited in this study, the mean age was $70.5 \pm 7.5$ (mean $\pm \mathrm{SD}$ ) years, and the median age (25th percentile, 75 th percentile) was 69.0 $(64.0,76.0)$ years with ranges from 60 to 97 years. Among the 1984 participants, there were 940 men $(47.4 \%)$ and 1044 women $(52.6 \%)$, overall sex ratio (Men/Women) was 0.90 , age specific sex ratios were ranged from 0.66 to 1.15 (Table S2).

In general, static balance, postural stability, dynamic balance, and overall balance performance scores decreased gradually with age increases (all $P<0.0001$ ). Relative to levels of 60- years, postural stability and overall balance performance decreased to significant levels since 65 years old, static balance and dynamic balance capacities started to decrease significantly since 70 years old (Table 1). In the elderly, individual variations of domains static balance, postural stability, and dynamic balance together with balance performance were all increased gradually over age, and roughly coefficients of variations increased substantially started from 75 years old. Throughout varying age groups, static balance and dynamic balance showed larger variations than postural

Table 1 Balance performances and variations in the elderly by age

\begin{tabular}{|c|c|c|c|c|c|c|c|c|c|}
\hline \multirow[t]{2}{*}{ Age (yrs) } & \multirow[t]{2}{*}{$\mathrm{n}$} & \multicolumn{2}{|c|}{ Static balance } & \multicolumn{2}{|c|}{ Postural stability } & \multicolumn{2}{|c|}{ Dynamic balance } & \multicolumn{2}{|l|}{ Balance } \\
\hline & & Mean $\pm S D$ & CV (\%) & Mean $\pm S D$ & CV (\%) & Mean $\pm S D$ & CV (\%) & Mean $\pm S D$ & CV (\%) \\
\hline $60-$ & 511 & $3.76 \pm 0.76^{\mathrm{a}}$ & 20.1 & $7.70 \pm 0.90^{a}$ & 11.7 & $7.73 \pm 1.06^{\mathrm{a}}$ & 13.7 & $19.20 \pm 2.13^{a}$ & 11.1 \\
\hline $65-$ & 530 & $3.67 \pm 0.87^{a b}$ & 23.7 & $7.42 \pm 1.25^{b}$ & 16.8 & $7.54 \pm 1.32^{\mathrm{ab}}$ & 17.5 & $18.63 \pm 2.73^{b}$ & 14.7 \\
\hline 70- & 378 & $3.54 \pm 0.94^{b}$ & 26.7 & $7.28 \pm 1.36^{b}$ & 18.6 & $7.46 \pm 1.47^{b}$ & 19.7 & $18.27 \pm 3.09^{b}$ & 16.9 \\
\hline $75-$ & 297 & $3.24 \pm 1.21^{c}$ & 37.2 & $6.67 \pm 1.86^{c}$ & 27.9 & $6.85 \pm 2.24^{c}$ & 32.8 & $16.76 \pm 4.49^{c}$ & 26.8 \\
\hline 80- & 165 & $2.93 \pm 1.41^{\mathrm{cd}}$ & 48.2 & $6.22 \pm 2.02^{c}$ & 32.5 & $6.09 \pm 2.85^{d}$ & 46.7 & $15.24 \pm 5.39^{d}$ & 35.3 \\
\hline $85-97$ & 103 & $2.44 \pm 1.50^{d}$ & 61.4 & $5.11 \pm 2.50^{d}$ & 49.0 & $4.91 \pm 3.25^{e}$ & 66.2 & $12.46 \pm 6.57^{e}$ & 52.7 \\
\hline Total & 1984 & $3.48 \pm 1.07$ & 30.7 & $7.13 \pm 1.61$ & 22.5 & $7.21 \pm 1.91$ & 26.4 & $17.83 \pm 3.94$ & 22.1 \\
\hline F & & 47.38 & & 77.95 & & 64.38 & & 91.78 & \\
\hline$P$ & & $<0.0001$ & & $<0.0001$ & & $<0.0001$ & & $<0.0001$ & \\
\hline
\end{tabular}

Superscript lowercase letters ( $a, b, c$, etc) indicated multiple comparison results among various age groups. Same letters indicated non-significant difference, different letters indicated significant differences in statistics. Significance level was 0.05 . Coefficients of variation (CV) was in percent 


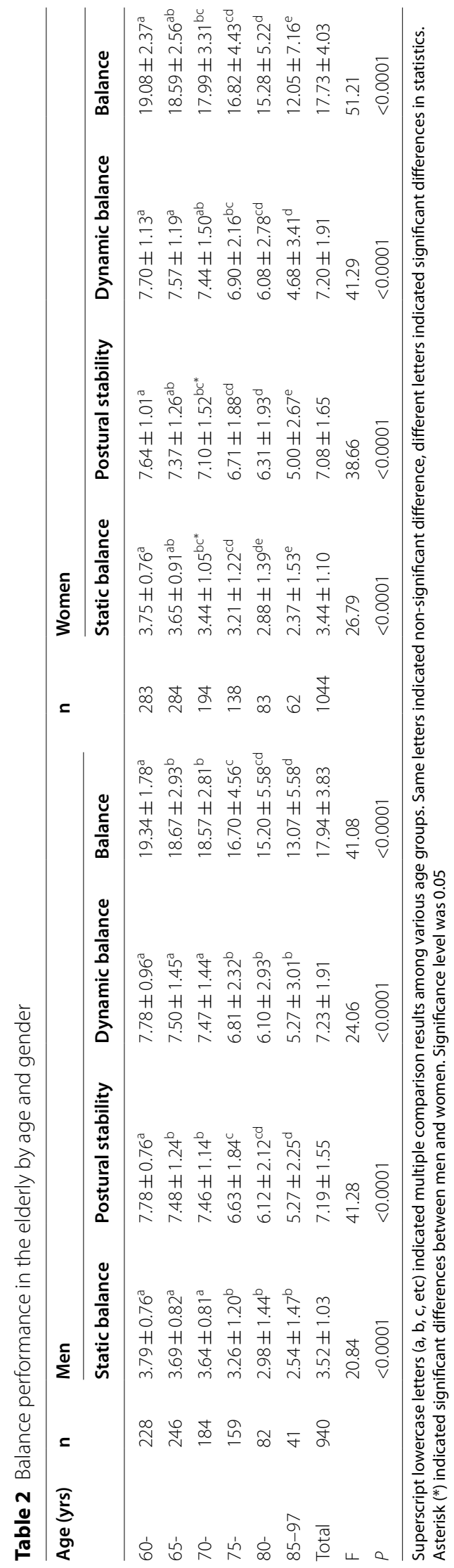


stability. At 85-97years, coefficients of variations for static balance and dynamic balance were $61.4 \%$ and $66.2 \%$, respectively, while that for postural stability was 49.0\% (Table 1).

There was no significant difference in each balance domain and overall balance performance between men and women. However, at specific age groups, there were slight differences in balance capacities and descending trends between men and women. At 70- years, static balance and postural stability scores in men were significantly higher than women $(P<0.05$ and $P<0.01$, respectively), balance performance score in men was marginally significantly higher than women $(P=0.067)$. In men, static and dynamic balance scores started to decrease significantly since 75 years old, whereas postural stability and overall balance performance started to decrease since 65 years. In women, dynamic scores started to decrease significantly since 75 years old, while static balance, postural stability, and overall balance performance started to significantly decrease since 70 years (Table 2 ).

In terms of descending rate, each balance domain and overall balance performance showed similar patterns. Overall, declines in balance performances were getting faster and faster with increases of age. Before 75 years old, declines were relatively slow, during 70 to 85 years declines were approximately linear, and after 85 years old, declines were the steepest. There were slight differences in decline pattern between men and women. In general, static balance in men was better than women across all ages. During 65 years to 75 years, in men static balance, postural stability, and dynamic balance all maintained at relatively stable level, however in women the decreases in static balance and postural stability were observable. Since 80 years old, declines in each balance domain and overall balance performance in men were less than women, and after 85 years old each balance domain and overall balance performance in men were better than women (Fig. 1).

Tasks in static balance was evaluated. Before 75 years old, over $90 \%$ individuals were able to perform tasks 1,2 , and 3 successfully. After 75 years old, performances for tasks 1,2, and 3 started to decrease gradually. Across all age groups, portions of individuals able to perform task 4 successfully were significantly lower than portions for tasks 1, 2, and 3. For task 4, in 60- years $88.8 \%$ individuals were able to perform successfully, in 70- years and 80years the portions were $75.9 \%$ and $55.8 \%$, respectively. And the portion was down to $35.9 \%$ in individuals aged 85 years and over whereas the portions were $60.2 \%$ to $75.7 \%$ for tasks 1, 2, and 3 (Table 3). Men and women had similar patterns (Table S3).

For tasks 5 and 6 in postural stability, there was no significant decrease in performance from 60 to 75 years old, over $90 \%$ individuals were able to perform successfully cross these 3 age groups. In 75- years, the portions substantially decreased to $78.8 \%$ and $74.7 \%$, respectively. And after 85 years, the portions were down to $49.5 \%$ and $42.7 \%$, respectively. In general performances for task 5 and task 6 in the elderly were significantly better than tasks 7 and 8 . Success portions for tasks 5 and 6 were $87.0 \%$ and $84.7 \%$, respectively, while success portions for tasks 7 and 8 were $76.1 \%$ and $72.8 \%$, respectively. Before 85 years old portions of successful performances for tasks 5 to 6 were significantly higher than portions for tasks 7 to 8 . There was no significant difference in portions between items over 85 years old (Table 4). Men and women had similar patterns (Table S4 and S5).

Among 8 tasks in dynamic balance, before 75 years old, over $90 \%$ individuals were able to perform tasks 9 , $10,12,13,14,15$, and 16 successfully. At 80 - years old, more than $80 \%$ elderly were able to perform tasks 14 and 15 successfully. Performances for task 11 was the worst, since 65 years old portions with success performance were below $90 \%$, till over 85 years old the portion was down to $49.5 \%$ while portions for other tasks were ranged from $56.3 \%$ to $72.8 \%$ (Table 5). Men and women had similar trends across ages (Table S6 and S7).

A two-step cluster analysis was applied to identify group segmentations, balance performances were categorized into 3 groups with scores of 0 to 10,11 to 17 , and 18 to 20 , which were arbitrarily named poor, fair, and good, respectively. With increases of age, proportions with poor and fair balance capacities elevated gradually, and after 85 years old reached up to $36.9 \%$ and $33.0 \%$, respectively (Table 6). There was slight difference in increasing pattern between men and women. In men the proportion with poor balance capacity increased from $12.6 \%$ at 75 years old to $29.3 \%$ at $85-97$ years old, while in women the portion elevated substantially from $8.0 \%$ to $41.9 \%$ during corresponding age stages (Table S8).

\section{Discussion}

The present study provided detailed reference information on balance performances in community elderly population. Results demonstrated the subjects with older ages had lower scores for overall balance performance, separate domains, and specific tasks. With increases of age, the proportions of the elderly with impaired balance increased stably and substantially.

Based on these age and gender specific data information on balance performance resulted from this study, in research and geriatric assessment settings, subpopulation and high risk groups with and at risk of specific domain or item impairments at certain age and gender would be able to be identified, then further examinations followed by targeted interventions would be possible and feasible 

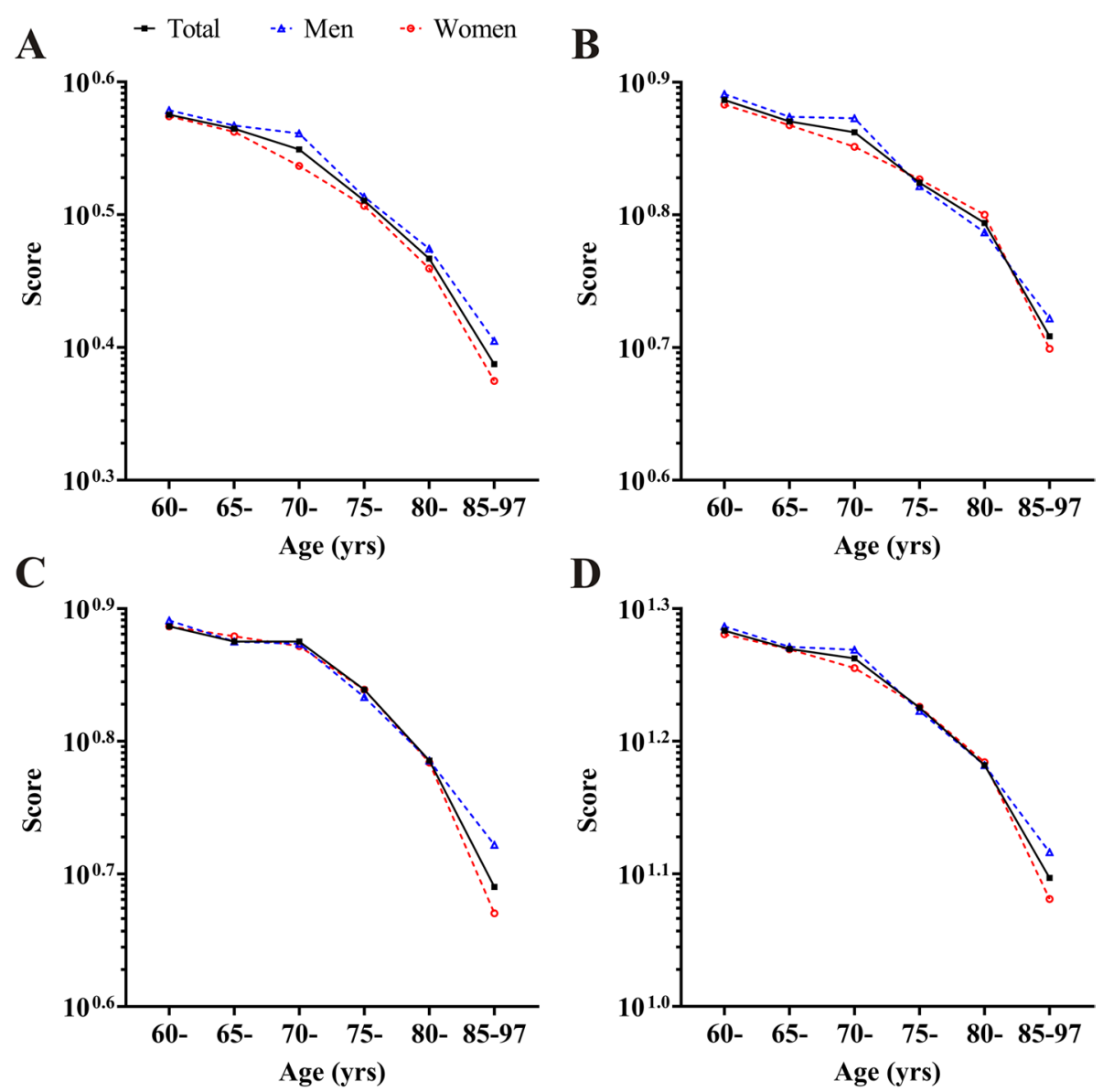

Fig. 1 Decline rates of balance performances in the elderly by age. A Static balance. B Postural stability. C Dynamic balance. D Balance performance

Table 3 Static balance in the elderly by age

\begin{tabular}{|c|c|c|c|c|c|c|c|c|c|}
\hline \multirow[t]{2}{*}{ Age (yrs) } & \multirow[t]{2}{*}{$n$} & \multicolumn{2}{|l|}{11} & \multicolumn{2}{|l|}{12} & \multicolumn{2}{|l|}{13} & \multicolumn{2}{|l|}{14} \\
\hline & & $n$ & $\%$ & $n$ & $\%$ & $n$ & $\%$ & $n$ & $\%$ \\
\hline 60- & 511 & 490 & $95.9^{\mathrm{a}}{ }_{\mathrm{A}}$ & 492 & $96.3^{\mathrm{a}}{ }_{\mathrm{A}}$ & 487 & $95.3^{\mathrm{a}}{ }_{\mathrm{A}}$ & 454 & $88.8^{\mathrm{a}}{ }_{B}$ \\
\hline 65- & 530 & 502 & $94.7^{a b}$ & 500 & $94.3^{\mathrm{a}} \mathrm{A}$ & 498 & $94.0^{\mathrm{a}}{ }_{\mathrm{A}}$ & 446 & $84.2^{\mathrm{a}}{ }_{B}$ \\
\hline 70- & 378 & 352 & $93.1^{\mathrm{ab}}{ }_{\mathrm{A}}$ & 356 & $94.2^{\mathrm{ab}}{ }_{\mathrm{A}}$ & 342 & $90.5^{\mathrm{ab}}{ }_{\mathrm{A}}$ & 287 & $75.9^{b}{ }_{B}$ \\
\hline 75- & 297 & 266 & $89.6^{b c}{ }_{A}$ & 261 & $87.9^{b c}$ & 248 & $83.5^{\mathrm{bc}} \mathrm{A}$ & 187 & $63.0^{C}{ }_{B}$ \\
\hline 80- & 165 & 138 & $83.6^{\mathrm{cd}}{ }_{\mathrm{A}}$ & 132 & $80.0^{\mathrm{cd}}{ }_{\mathrm{A}}$ & 121 & $73.3^{\mathrm{cd}}{ }_{\mathrm{A}}$ & 92 & $55.8^{C}{ }_{B}$ \\
\hline $85-97$ & 103 & 78 & $75.7_{A}^{d}$ & 74 & $71.8^{\mathrm{d}}{ }_{\mathrm{A}}$ & 62 & $60.2^{d}{ }_{A}$ & 37 & $35.9^{d}{ }_{B}$ \\
\hline Total & 1984 & 1826 & $92.0_{\mathrm{A}}$ & 1815 & $91.5_{\mathrm{A}}$ & 1758 & $88.6_{B}$ & 1503 & $75.8_{C}$ \\
\hline$x^{2}$ & & 71.9 & & 108.0 & & 167.3 & & 219.4 & \\
\hline$P$ & & $<0.0001$ & & $<0.0001$ & & $<0.0001$ & & $<0.0001$ & \\
\hline
\end{tabular}

Superscript lowercase letters (a, b, c, etc) indicated multiple comparison results among various age groups. Subscript capital letters (A, B, C, etc) indicated multiple comparison results between items. Same letters indicated non-significant difference, different letters indicated significant differences in statistics. Significance level was 0.05 


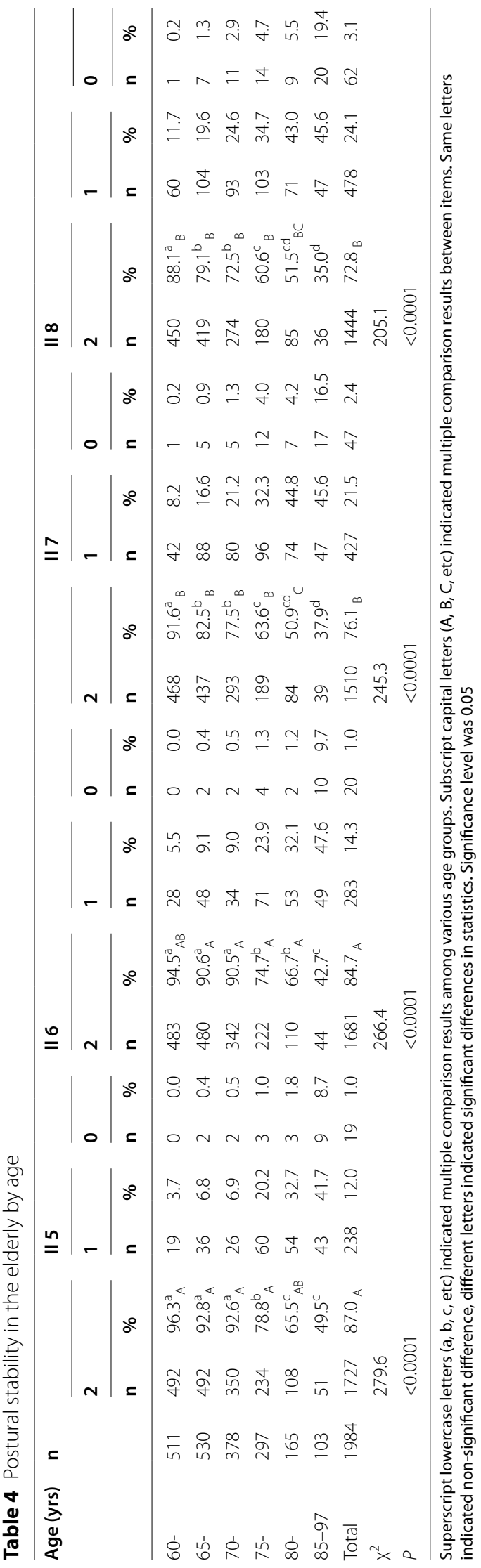




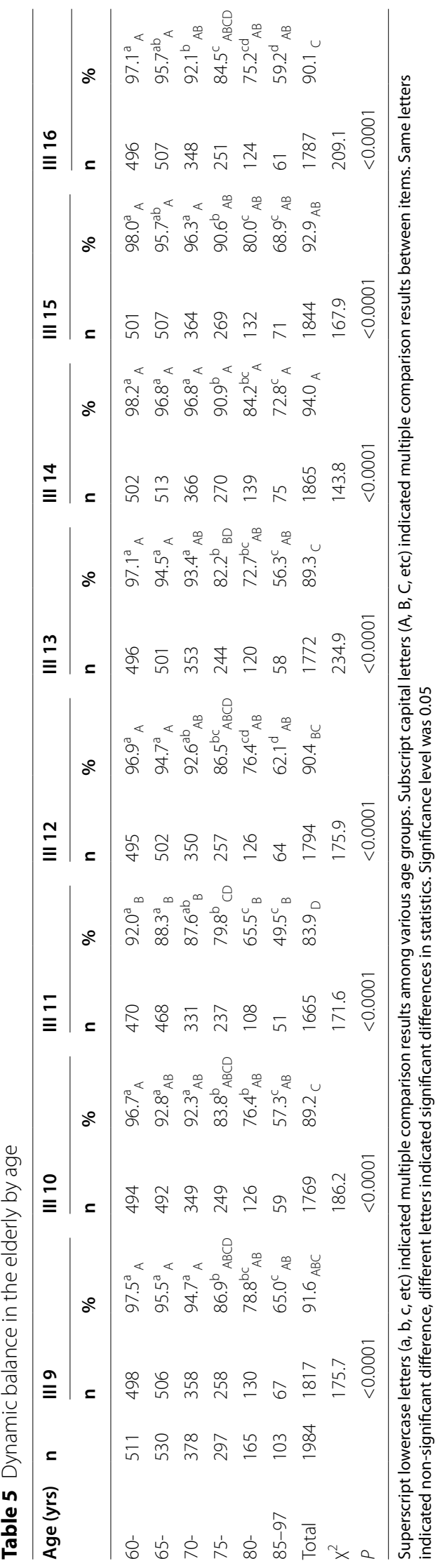


Table 6 Categories of balance performance in the elderly by age

\begin{tabular}{|c|c|c|c|c|c|c|c|}
\hline \multirow[t]{2}{*}{ Age (yrs) } & \multirow[t]{2}{*}{$\mathbf{n}$} & \multicolumn{2}{|c|}{ Poor (0-10) } & \multicolumn{2}{|c|}{ Fair (11-17) } & \multicolumn{2}{|c|}{ Good (18-20) } \\
\hline & & $\mathrm{n}$ & $\%$ & $\mathrm{n}$ & $\%$ & $\mathrm{n}$ & $\%$ \\
\hline 60- & 511 & 8 & $1.6^{\mathrm{a}}$ & 42 & $8.2^{\mathrm{a}}$ & 461 & $90.2^{\mathrm{a}}$ \\
\hline 65- & 530 & 13 & $2.5^{\mathrm{a}}$ & 84 & $15.8^{b}$ & 433 & $81.7^{b}$ \\
\hline 70- & 378 & 18 & $4.8^{\mathrm{ab}}$ & 66 & $17.5^{b c}$ & 294 & $77.8^{b}$ \\
\hline 75- & 297 & 31 & $10.4^{b}$ & 82 & $27.6^{d}$ & 184 & $62.0^{\circ}$ \\
\hline 80- & 165 & 37 & $22.4^{c}$ & 47 & $28.5^{c d}$ & 81 & $49.1^{\circ}$ \\
\hline $85-97$ & 103 & 38 & $36.9^{c}$ & 34 & $33.0^{d}$ & 31 & $30.1^{\circ}$ \\
\hline Total & 1984 & 145 & 7.3 & 355 & 17.9 & 1484 & 74.8 \\
\hline
\end{tabular}

A two-step cluster analysis was applied to identify group segmentations. Superscript lowercase letters (a, b, c, etc) indicated multiple comparison results among various age groups. Same letters indicated non-significant difference, different letters indicated significant differences in statistics. Significance level was 0.05

in clinics and public health to improve their balance performance.

In the present study sex ratio of the elderly population aged 60 years and above was within ranges of ratios in China and worldwide, the sex ratio aged 85 years and over was also comparable to the global ratio. In contrast to overall sex ratio of elderly aged 60 years and over, sex ratio of the elderly aged 85 years and above was obviously lower, this pattern was in agreement with data in China and worldwide as well. Women have longer life expectancies than men in world, China, and Shanghai, which is attributable to lower men to women sex ratio in the oldest elderly at least in part $[2,18-21]$.

To elucidate characters of balance performance with changes of age, subjects were classified into specific age groups with a 5 -year interval. There were relatively limited individuals aged 85 years or over, therefore they were combined within 1 group, making a total of 6 age groups for analytic comparisons. The 16 items of X16 balance testing scale were clustered into 3 domains of static balance, postural stability, and dynamic balance [17]. Thus balance performances in the elderly were elucidated in details at levels of total balance performance, separate domains, and individual items.

Elucidations of balance performance stratified by age and gender provided valuable evidence for references and interventions. In the elderly static balance, postural stability, dynamic balance, and overall balance performance were compared between age groups. As expected, in general balance scores decreased gradually with age increase. However, relative to declines in static balance and dynamic balance starting from 70 years old, postural stability started to worsen since 65 years, making overall balance performance started to decrease at 65 years. After 80 years old, both overall balance performance and individual domains started to deteriorate substantially in both men and women. These findings provided of value scientific information for guidance on timely and well focused screenings, examinations, and interventions accordingly. The signature benefits may be aimed policy programs on subpopulations with certain levels of functioning, which would allow for a significantly reduced sample size.

In this study there was no significant difference observed in static balance, postural stability, dynamic balance, or overall balance performance between men and women. Yet in the elderly aged 85 years and over, men had somewhat slightly higher scores in static balance, postural stability, dynamic balance, and overall balance performance than women. Previous studies showed inconsistent results. For example, a report from community dwelling elderly individuals in Brazil found there was no significant difference in scores on Berg Balance Scale regarding gender [22]. While a study based on a Turkish sample showed men had significantly better balance performance in comparison to women on functional reach test, the timed up and go test, the sit to stand test, and the step test in participants aged 50 to 75 years old [10]. And a study based on Danish population showed the elderly men performed worse than the elderly women in static balance [11]. A study in Finnish population elucidated effects of age, gender and their interactions on postural balance with various tests, confirmed properties of age and gender dependency [12]. Thus it could be concluded these conflicting findings could be due to contributions of ethnicities, population features, ages, measurement approaches and others.

The present results demonstrated individual variations in static balance, postural stability, dynamic balance, and overall balance performance all increased gradually and substantially over ages in the elderly. Balance performance requires profound integration of vision, vestibular sense, proprioception, muscle strength, neuromuscular, and skeletal function. With increased age, progressive dysfunctions on these systems would result in balance deficits. Where diversities 
in these functional disorders over age and cumulative impacts of these health inequities across life course would contribute to larger varieties in balance performance [23, 24]. Previous study showed variability in gait velocity increased in older adults compared to young and middle-aged adults, which is in line with the present findings of increasing variations across ages [25].

It was not a surprise that portions with success performance on specific tasks declined gradually with increases in age. However, there were differences in deterioration extent between tasks. In static balance, portions with success performance on 3 tasks of standing on two legs were comparable. Whereas relative to these 3 tasks, portions with success performance on standing on one leg (item 4) was substantially lower across all age groups, these obvious worsening abilities of standing on one leg hinted significantly reduced balance of dominant leg at functional level started from 60 years old and earlier.

Amongst the 4 tasks of postural stability, portions with success performance on postural transfers between standing and squatting were lower than transfers between standing and sitting, and portions with intact abilities of transferring from squatting to standing (item 8) were the lowest. Achievements of these postural transfers depend largely on strengths of lower body extremities which were reduced gradually with advances in age. The worsening severity of balance performance on specific task could be attributable to extents of dependencies on and deterioration of strengths of lower body extremities [26].

Among the 8 tasks of dynamic balance, portions of the elderly with normal step length (item 11) was the lowest in the elderly. Previous studies had revealed significant decreases in step length over ages in the elderly. The elderly tended to develop a more cautious gait which was characterized by a reduced step length consequently a reduced gait speed [27].

Collectively, the performance on standing on one leg and normal step length decreased distinctively since 70 years old, and transfers between squatting and standing postures decreased obviously as early as 65 years old, suggesting preventive and therapeutic measures aiming to improve these abilities are in urgent needs.

Given desirable features of handling categorical and continuous variables and automatic selection of number of clusters, the two-step cluster analysis was applied in this research to identify group segmentations.

The present study was based on community setting population who are functionally independent, thus the findings may not be generalized to populations with requirements for nursing or hospitalization. The X16 scale was designated for screening for high risk subpopulation from community setting or large scale study, selected individuals subject to further examination and diagnosis for subsequent treatment and intervention.

Theoretically, it was possible that the Hawthorne effect [28] might exist in the study, making the observed balance performance from the participants might be slightly better than the realities in the population.

The previous results demonstrated that both the reliability and validity of the X16 scale were adequate and acceptable [17], and difficulties of the task were taken into consideration for the level rating. However, due to practical difficulties the weights of each item have not been evaluated more exactly. The calculation of the total balance score may subject to optimization upon availability of appropriate weighting solution.

\section{Conclusions}

In the elderly, with advances in age, abilities of overall balance performance, individual domains of static balance, postural stability, and dynamic balance, and successful performances on specific tasks declined gradually and stably. The deterioration started to be obvious since 65-75 years. Performances on standing on one leg, postural transfering from squatting to standing, and step length was worse than remaining tasks in their corresponding domains of static balance, postural stability, and dynamic balance, respectively. Men and women had similar patterns.

\section{Supplementary Information}

The online version contains supplementary material available at https://doi. org/10.1186/s12877-021-02560-9.

Additional file 1: Table S1 The 16 items of the X16 balance testing scale for the elderly. Table S2 Age and gender compositions of the elderly. Table S3 Static balance in the elderly by age and gender. Table S4 Postural stability in the elderly men by age. Table S5 Postural stability in the elderly women by age. Table S6 Dynamic balance in the elderly men by age. Table S7 Dynamic balance in the elderly women by age. Table $\mathbf{8 8}$ Categories of balance performance in the elderly by age and gender.

\section{Acknowledgments}

We would like to express our appreciations to all the subjects for their participating the study. We also greatly appreciate the contributions to field investigations from the staff of Community Health Centers in the Changning District, Shanghai

\section{Authors' contributions}

Study conceiving and design, YJ, QX. Investigation conduct, PZ, YJ, QX. Data entry and processing, $X L, Y F, Z Y, P Z, X Y, Y J, Q X$. Data analysis and data interpretation, HW, YW, XM, XL, YF, ZY, XY, QX. Manuscript draft, HW, YW, XM, JZ, QX. Manuscript revision, HW, JZ, QX. All authors reviewed and approved the final manuscript

\section{Funding}

The work was supported by Research Programs for Higher Education and Teaching in Wenzhou Medical University (YBJG2019007), Research Programs of Wenzhou Medical University for Undergraduates (Natural Science wyx2020101043), and Training Programs of Innovation and Entrepreneurship for Undergraduates at School of Public Health and Management of Wenzhou 
Medical University. The funding body was not involved in the study design, data interpretation, or manuscript writing.

\section{Availability of data and materials}

The data used in the study are available from the corresponding author on reasonable request.

\section{Declarations}

Ethics approval and consent to participate

The project was approved by Institutional Review Board (IRB) of Changning Center for Disease Control and Prevention, Shanghai. Written informed consents were obtained.

\section{Consent for publication}

Not applicable.

\section{Competing interests}

The authors declare that they have no competing interests.

\section{Author details}

${ }^{1}$ Department of Preventive Medicine, Institute of Nutrition and Diseases, Wenzhou Medical University, Wenzhou 325035, People's Republic of China. ${ }^{2}$ Department of Traditional Chinese Medicine, Shanghai University of Traditional Chinese Medicine, Shanghai 201203, People's Republic of China. ${ }^{3}$ School of Medicine, Hunan Normal University, Changsha 410013, People's Republic of China. ${ }^{4}$ National Center for AIDS/STD Control and Prevention, Chinese Center for Disease Control and Prevention, Beijing 102206, People's Republic of China. ${ }^{5}$ Changning Center for Disease Control and Prevention, Shanghai 200051, People's Republic of China.

Received: 21 April 2021 Accepted: 13 October 2021

Published online: 25 October 2021

\section{References}

1. United Nations, Department of Economic and Social Affairs, Population Division. World population ageing 2019: highlights. New York: the United Nations; 2019.

2. Guzmán JM, Pawliczko A, Beales S, Till C, Voelcker I. Ageing in the twentyfirst century:a Celebration and a challenge. New York: United Nations Population Fund (UNFPA); 2012.

3. Abellan van Kan G, Rolland Y, Andrieu S, Bauer J, Beauchet O, Bonnefoy $M$, et al. Gait speed at usual pace as a predictor of adverse outcomes in community-dwelling older people an international academy on nutrition and aging (IANA) task force. J Nutr Health Aging. 2009;13(10):881-9.

4. Montero-Odasso M, Schapira M, Soriano ER, Varela M, Kaplan R, Camera $L A$, et al. Gait velocity as a single predictor of adverse events in healthy seniors aged 75 years and older. J Gerontol: Med Sci. 2005;60(10):1304-9.

5. Pollock AS, Durward BR, Rowe PJ, Paul JP. What is balance? Clin Rehabil. 2000;14:402-6.

6. Berg K. Measuring balance in the elderly: preliminary development of an instrument. Physiother Can. 1989;41(6):304-11.

7. Horak FB. Clinical measurement of postural control in adults. Phys Ther. 1987;67:1881-5.

8. Winter DA, Patla AE, Frank JS. Assessment of balance control in humans. Med Prog Technol. 1990;16:31-51.

9. King MB, Judge JO, Wolfson L. Functional base of support decreases with age. J Gerontol: Med Sci. 1994;49(6):M258-63.

10. Aslan UB, Cavlak U, Yagci N, Akdag B. Balance performance, aging and falling: a comparative study based on a Turkish sample. Arch Gerontol Geriatr. 2008;46(3):283-92.
11. Riis J, Eika F, Blomkvist AW, Rahbek MT, Eikhof KD, Hansen MD, et al. Lifespan data on postural balance in multiple standing positions. Gait Posture. 2020;76:68-73.

12. Era P, Sainio P, Koskinen S, Haavisto P, Vaara M, Aromaa A. Postural balance in a random sample of 7,979 subjects aged 30 years and over. Gerontology. 2006;52(4):204-13.

13. Leirós-Rodríguez R, Romo-Pérez V, García-Soidán JL, García-Liñeira J. Percentiles and reference values for the accelerometric assessment of static balance in women aged 50-80 years. Sensors. 2020;20(3):940.

14. Verghese J, LeValley A, Hall CB, Katz MJ, Ambrose AF, Lipton RB. Epidemiology of gait disorders in community-residing older adults. J Am Geriatr Soc. 2006;54(2):255-61.

15. Downs S, Marquez J, Chiarelli P. Normative scores on the Berg balance scale decline after age 70 years in healthy community-dwelling people: a systematic review. J Phys. 2014;60(2):85-9.

16. Franchignoni F, Horak F, Godi M, Nardone A, Giordano A. Using psychometric techniques to improve the balance evaluation systems test: the mini-BESTest. J Rehabil Med. 2010;42(4):323-31.

17. Ju J, Jiang Y, Zhou P, Li L, Ye X, Wu H, et al. Evaluation of the reliability and validity for X16 balance testing scale for the elderly. BMC Geriatr. 2018;18(1):112.

18. World health statistics 2020. Monitoring health for the SDGs, sustainable development goals. Geneva: World Health Organization; 2020.

19. National Bureau of Statistics of China. China statistical yearbook. Beijing: China Statistics Press; 2019.

20. Shanghai Municipal Statistics Bureau, Survey Office of the National Bureau of Statistics in Shanghai. Shanghai statistical yearbook. China academic journal electronic publishing house; 2019.

21. United Nations, Department of Economic and Social Affairs, Population Division. World population ageing 1950-2050. New York: the United Nations; 2002.

22. Nakagawa HB, Ferraresi JR, Prata MG, Scheicher ME. Postural balance and functional independence of elderly people according to gender and age: cross-sectional study. Sao Paulo Med J. 2017;135(3):260-5.

23. Sturnieks DL, St George R, Lord SR. Balance disorders in the elderly. Neurophysiologie clinique/Clin Neurophysiol. 2008;38(6):467-78.

24. Skelton DA, Mavroeidi A. How do muscle and bone strengthening and balance activities (MBSBA) vary across the life course, and are there particular ages where MBSBA are most important? J Frailty Sarcopenia Falls. 2018;03(02):74-84

25. Hollman JH, Kovash FM, Kubik JJ, Linbo RA. Age-related differences in spatiotemporal markers of gait stability during dual task walking. Gait Posture. 2007;26(1):113-9.

26. Wiacek M, Hagner W, Hagner-Derengowska M, Bluj B, Drozd M, Czereba J, et al. Correlations between postural stability and strength of lower body extremities of women population living in long-term care facilities. Arch Gerontol Geriatr. 2008;48(3):346-9.

27. Herssens N, Verbecque E, Hallemans A, Vereeck L, Van Rompaey V, Saeys W. Do spatiotemporal parameters and gait variability differ across the lifespan of healthy adults? A systematic review. Gait Posture. 2018;64:181-90.

28. McCambridge J, Witton J, Elbourne DR. Systematic review of the Hawthorne effect: new concepts are needed to study research participation effects. J Clin Epidemiol. 2014;67(3):267-77.

\section{Publisher's Note}

Springer Nature remains neutral with regard to jurisdictional claims in published maps and institutional affiliations. 